\title{
Multifocal Jejuno-ileal carcinoma in a 7-year-old boy with Peutz-Jeghers syndrome: A rare occurence
}

Sir,

Peutz-Jeghers syndrome (PJS) is a rare disease with incidence of about 1 in 50000-200000 live births and was described independently by Peutz in 1921 and Jeghers in 1949. The patients with PJ syndrome have elevated risk of gastrointestinal and extra-gastrointestinal malignancies which includes breast and ovarian cancers in women, testicular tumors in males and cancers of the pancreas, esophagus, stomach and lung in both sexes. The cumulative risk increases with age. ${ }^{[1]}$

Our patient is a 7-year-old boy who, while being investigated for aortic stenosis was noticed to have pigmentation in the palms, soles and perioral region. With clinical suspicion of PJS he was advised upper gastrointestinal endoscopy, which showed multiple polyps in the jejunum and ileum. Colonoscopy and computed tomography abdomen were within normal limits. He was on follow-up for 2 years and was asymptomatic. He presented to the surgical emergency ward with colicky abdominal pain, vomiting and blood in stools over previous 2 days. Clinical examination suggested intestinal obstruction and surgery was performed. Three specimens labeled as "ileum," "jejunal tissue" and "multiple polyps" were sent to histopathology laboratory. Jejunum showed multiple polyps, the largest measuring $2.5 \mathrm{~cm}$ in diameter and ileum had a single large polyp of $2 \mathrm{~cm}$ diameter [Figure 1a]. On microscopy, polyps in both jejunum and ileum showed dysplastic glands infiltrating transmurally up to the serosa. The glands were lined by stratified cells with a moderate amount of cytoplasm and hyperchromatic nuclei and a diagnosis of well-differentiated adenocarcinoma of the jejunum and ileum was made. The specimen sent as "multiple polyps" showed features of a hamartomatous polyp characterized by arborizing musculature and hyperplastic glands [Figure 1b-d].

The diagnostic criterion for PJS was proposed by Giardello et al. ${ }^{[1]}$ and requires histopathological confirmation of hamartomatous gastrointestinal polyps with any two of the following features:

a. Small bowel polyposis

b. Family history and

c. Pigmented skin and/or mucosal brown macules. ${ }^{[2]}$

Although hamartomatous polyps commonly involve the jejunum, colon is the most common site for gastrointestinal cancer. ${ }^{[3]}$ Carcinoma of the jejunum and ileum are distinctively rare and multifocality has not been

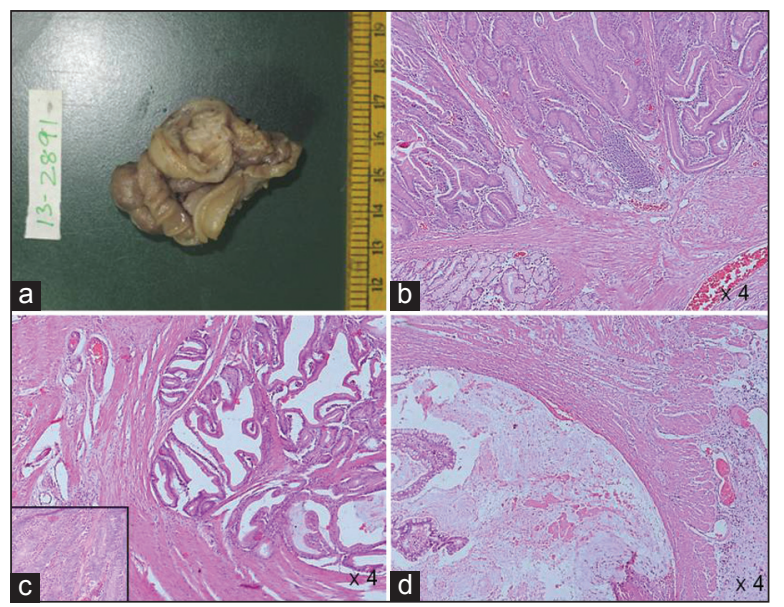

Figure 1: (a) Segment of the small intestine with polyp (b) Smaller separately lying polyps show typical features of an hamartomatous polyp with arborizing smooth muscle. (c) Carcinomatous areas show invasion of dysplastic glands in to the muscle layer with glandular epithelium showing features of high grade dysplasia (inset) along with (d) Dissecting type of extracellular mucin extending to the serosal layer

reported till date. The incidence of adenomatous and carcinomatous transformation in hamartomatous polyps is about $6-12 \%$. Great care has to be taken in diagnosing carcinoma in hamartomatous polyp as frequent mitosis, occasional hyperchromatic nucleus, low grade dysplasia and pseudo invasion commonly occurs in PJ polyps. A clear cut invasion by dysplastic glands, cytological features of malignancy, reaction around the infiltrating glands, dissecting pools of mucin and metastasis to distant sites are prerequisite for diagnosis of malignancy in PJS.

The average age of developing malignancies was found to be 23.5 years in a Korean study done on 30 patients of PJS..$^{[4]}$ Mehta et al. ${ }^{[5]}$ reported a case of intestinal adenocarcinoma with PJS in a 25 -year-old man while Saranrittichai reported a case of PJS and colon cancer in a 10-year-old girl. ${ }^{[6]}$ Recently, intraoperative endoscopy and endoscopic polypectomy, rather than segmental resections of the bowel, have been recommended as a method of managing PJS polyps. The present case is peculiar as it demonstrates one of the rare sites for carcinomas in patient with PJS, multifocality of the malignancy and occurrence at a very young age.

\section{Richa Ranjan, Devajit Nath, Santosh Dey', Sudheer Arava}

Departments of Pathology, and ${ }^{1}$ Pediatric Surgery, All India Institute of Medical Sciences, New Delhi, India. E-mail: aravaaiims@gmail.com 


\section{REFERENCES}

1. Giardiello FM, Welsh SB, Hamilton SR, Offerhaus GJ, Gittelsohn AM, Booker SV, et al. Increased risk of cancer in the Peutz-Jeghers syndrome. N Engl J Med 1987;316:1511-4.

2. Hearle N, Schumacher V, Menko FH, Olschwang S, Boardman LA, Gille JJ, et al. Frequency and spectrum of cancers in the PeutzJeghers syndrome. Clin Cancer Res 2006;12:3209-15.

3. Pan SY, Morrison H. Epidemiology of cancer of the small intestine. World J Gastrointest Oncol 2011;3:33-42.

4. Choi HS, Park YJ, Youk EG, Yoon KA, Ku JL, Kim NK, et al. Clinical characteristics of Peutz-Jeghers syndrome in Korean polyposis patients. Int J Colorectal Dis 2000;15:35-8.

5. Mehta MV, Porecha MM, Mehta PJ. Small intestinal adenocarcinoma in Peutz-Jeghers syndrome. Indian J Gastroenterol 2006;25:38-9.

6. Saranrittichai S. Peutz-Jeghers syndrome and colon cancer in a 10-year-old girl: Implications for when and how to start screening? Asian Pac J Cancer Prev 2008;9:159-61.

\begin{tabular}{|l|l|}
\hline \multicolumn{2}{|c|}{ Access this article online } \\
\hline Quick Response Code: & Website: \\
\hline & www.ijmpo.org \\
\hline & \\
\hline
\end{tabular}

